\title{
Features of 20133 UK patients in hospital with covid-19 using the ISARIC WHO Clinical Characterisation Protocol: prospective observational cohort study
}

Annemarie B Docherty, ${ }^{1,2}$ Ewen M Harrison, ${ }^{1}$ Christopher A Green, ${ }^{3}$ Hayley E Hardwick, ${ }^{4,5}$ Riinu Pius, ${ }^{1}$ Lisa Norman, ${ }^{1}$ Karl A Holden, ${ }^{6}$ Jonathan M Read, ${ }^{7}$ Frank Dondelinger, ${ }^{7}$ Gail Carson, ${ }^{8}$ Laura Merson, ${ }^{8,9}$ James Lee, ${ }^{8}$ Daniel Plotkin, ${ }^{8}$ Louise Sigfrid, ${ }^{8}$ Sophie Halpin, ${ }^{10}$ Clare Jackson, ${ }^{10}$ Carrol Gamble, ${ }^{10}$ Peter W Horby, ${ }^{11}$ Jonathan S Nguyen-Van-Tam, ${ }^{12}$ Antonia Ho, ${ }^{13}$ Clark D Russell, ${ }^{14}$ Jake Dunning, ${ }^{15,16}$ Peter JM Openshaw, ${ }^{17}$ J Kenneth Baillie, ${ }^{2,18}$ Malcolm G Semple, ${ }^{19,20}$ on behalf of the ISARIC4C investigators

For numbered affiliations see end of the article.

Correspondence to: M G Semple m.g.semple@liverpool.ac.uk (or @TweedieChap on Twitter: ORCID 0000-0001-9700-0418) Additional material is published online only. To view please visit the journal online.

Cite this as: $B M J$

2020;369:m1985

http://dx.doi.org/10.1136/ bmj.m1985

Accepted: 15 May 2020

\section{ABSTRACT}

OBJECTIVE

To characterise the clinical features of patients admitted to hospital with coronavirus disease 2019 (covid-19) in the United Kingdom during the growth phase of the first wave of this outbreak who were enrolled in the International Severe Acute Respiratory and emerging Infections Consortium (ISARIC) World Health Organization (WHO) Clinical Characterisation Protocol UK (CCP-UK) study, and to explore risk factors associated with mortality in hospital.

DESIGN

Prospective observational cohort study with rapid data gathering and near real time analysis.

SETTING

208 acute care hospitals in England, Wales, and Scotland between 6 February and 19 April 2020. A case report form developed by ISARIC and WHO was used to collect clinical data. A minimal follow-up time of two weeks (to 3 May 2020) allowed most patients to complete their hospital admission.

PARTICIPANTS

20133 hospital inpatients with covid-19.

MAIN OUTCOME MEASURES

Admission to critical care (high dependency unit or intensive care unit) and mortality in hospital.

\section{WHAT IS ALREADY KNOWN ON THIS TOPIC}

Observational studies in China have reported risk factors associated with severe covid-19 that requires hospital admission

Studies describing the features and outcomes of patients with severe covid-19 who have been admitted to hospital in Europe are lacking

Older male adults, people with diabetes, hypertension, cardiovascular disease, or chronic respiratory disease are at greater risk of severe covid-19 that requires hospital admission and higher levels of care, and are at higher risk of death

\section{WHAT THIS STUDY ADDS}

This rapid prospective investigation of patients with covid-19 admitted to hospital in England, Wales, and Scotland showed that obesity, chronic kidney disease, and liver disease were also associated with increased hospital mortality Obesity is a major additional risk factor that was not highlighted in data from China

Severe covid-19 leads to a prolonged hospital stay and a high mortality rate; over a quarter of inpatients in this study had died at the time of reporting, and nearly a third remained in hospital

\section{RESULTS}

The median age of patients admitted to hospital with covid-19, or with a diagnosis of covid-19 made in hospital, was 73 years (interquartile range 58-82, range 0 -104). More men were admitted than women (men $60 \%, n=12068$; women $40 \%, n=8065$ ). The median duration of symptoms before admission was 4 days (interquartile range 1-8). The commonest comorbidities were chronic cardiac disease (31\%, $5469 / 17702)$, uncomplicated diabetes (21\%, $3650 / 17599)$, non-asthmatic chronic pulmonary disease (18\%, 3128/17634), and chronic kidney disease (16\%, 2830/17 506); 23\% (4161/18525) had no reported major comorbidity. Overall, $41 \%$ $(8199 / 20133)$ of patients were discharged alive, $26 \%$ (5165/20133) died, and 34\% (6769/20133) continued to receive care at the reporting date. $17 \%(3001 / 18183)$ required admission to high dependency or intensive care units; of these, $28 \%$ (826/3001) were discharged alive, 32\% (958/3001) died, and 41\% (1217/3001) continued to receive care at the reporting date. Of those receiving mechanical ventilation, $17 \%(276 / 1658)$ were discharged alive, 37\% (618/1658) died, and 46\% (764/1658) remained in hospital. Increasing age, male sex, and comorbidities including chronic cardiac disease, nonasthmatic chronic pulmonary disease, chronic kidney disease, liver disease and obesity were associated with higher mortality in hospital.

\section{CONCLUSIONS}

ISARIC WHO CCP-UK is a large prospective cohort study of patients in hospital with covid-19. The study continues to enrol at the time of this report. In study participants, mortality was high, independent risk factors were increasing age, male sex, and chronic comorbidity, including obesity. This study has shown the importance of pandemic preparedness and the need to maintain readiness to launch research studies in response to outbreaks.

STUDY REGISTRATION

ISRCTN66726260.

\section{Introduction}

The outbreak of disease caused by the novel severe acute respiratory syndrome coronavirus 2 (SARSCoV-2) was declared a pandemic by the World Health Organization on 11 March $2020 .^{1}$ The WHO situation 
report dated 30 April 2020 stated 3090445 people had confirmed coronavirus disease 2019 (covid-19) and 217769 people had died across the world. ${ }^{2}$

In the wake of the influenza A H1N1 pandemic (2009) and the emergence of Middle East respiratory syndrome coronavirus (2012), it was recognised that the effectiveness of a response to a future pandemic threat would critically depend on the speed and focus of that response. The United Kingdom set up and maintained a "sleeping" prepandemic suite of protocols, documents, and agreements in preparation for future outbreaks. The International Severe Acute Respiratory and emerging Infections Consortium (ISARIC) WHO Clinical Characterisation Protocol UK (CCP-UK) study was a core component of this portfolio. ${ }^{3}$ Further details about ISARIC WHO CCP-UK can be found at https://isaric4c.net and in the online supplement.

In response to the emergence of SARS-CoV-2 and its pandemic potential, the ISARIC WHO CCP-UK study was activated on 17 January 2020, in time to enrol the first wave of patients with covid-19 admitted to hospitals in England and Wales. The first confirmed patient with covid-19 in the UK was reported on 31 January 2020.

Hospital admission rates for patients with covid-19 have been difficult to estimate because rates depend on the prevalence of community testing and admission criteria, which vary between countries. However, an estimated one in 10 to one in five adults have illnesses of sufficient severity to warrant hospital admission. ${ }^{4}$ Patients have mostly been admitted with severe acute respiratory infection or severe acute respiratory syndrome according to the previous WHO case definitions. ${ }^{56}$ The provision of intensive care also varies between countries. Studies first from China, and more recently from Europe and the United States, have found rates of admission to intensive care range from $5 \%$ to $32 \% .^{78}$ Old age, chronic major comorbidity, and male sex have consistently been associated with increased mortality. ${ }^{9-12}$

In this first report of the ISARIC WHO CCP-UK study, we characterise the clinical features of patients admitted to hospital with covid-19 in England, Scotland, and Wales during the growth phase of the first wave of this outbreak, up to 19 April 2020. Future reports will include Northern Ireland. We describe all patient outcomes as known on 3 May 2020 and explore risk factors associated with mortality in hospital.

\section{Methods}

\section{Study design and setting}

The ISARIC WHO CCP-UK (National Institute for Health Research Clinical Research Network Central Portfolio Management System ID: 14152) study is an ongoing prospective cohort study in 208 acute care hospitals in England, Scotland, and Wales. The protocol (supplementary material 2), revision history, case report form (version 9.2; supplementary material 3), information leaflets, consent forms and details of the
Independent Data and Material Access Committee are available online. ${ }^{21}$

\section{Participants}

Inclusion criteria were people of all ages who were admitted to one of 208 acute care hospitals in England, Scotland, and Wales with proven or high likelihood of infection with a pathogen of public health interest, defined as SARS-CoV-2 for this event by Public Health England. Reverse transcriptase polymerase chain reaction was the only mode of testing available during the period of study. The decision to test was at the discretion of the clinician attending the patient, and not defined by protocol. The enrolment criterion "high likelihood of infection" reflects that a preparedness protocol cannot assume that a diagnostic test will be available for an emergent pathogen. Site training emphasises that only patients who tested positive for covid-19 were eligible for enrolment.

National guidance was provided by Public Health England and other UK public health agencies that advised who to test based on clinical case definitions for possible covid-19 (online supplement). We also included patients who had been admitted for a separate condition but had tested positive for covid-19 during their hospital stay. We collected additional biological samples for research purposes when consent was given (please see online supplement for details of consent procedures and biological samples). These samples are currently undergoing analysis and we will present the results when they become available. Patients were only enrolled during their index admission. We used three tiers in the ISARIC WHO CCP-UK protocol. Patients in tier 0 had clinical information from their routine health records uploaded into the case report form. Consent was not required for collection of depersonalised routine healthcare data for research in England and Wales. A waiver for consent was given by the Public Benefit and Privacy Panel in Scotland. Tier 1 and 2 of the protocol involve additional biological sampling for research purposes for which consent by, or assent for, participants was obtained.

\section{Data collection}

We collected baseline demographic data on a paper case report form (version 9.2; supplementary material 2) that was developed by ISARIC and WHO for use in outbreak investigations. Data were uploaded from admission, and usually before hospital episodes were complete, to a REDCap database (Research Electronic Data Capture, Vanderbilt University, US, hosted by University of Oxford, UK). We aimed to record measures of illness severity and routine blood test results at a minimum of four time points: day of hospital admission (day 1), day 3, day 6, day 9, and day of any admission to critical care. We recorded relevant treatments that patients received in hospital, level of care (ward based, high dependency unit, or intensive care unit), complications, and details of discharge or death while in hospital. Further information about these variables can be found in the online supplement. 


\section{Outcomes}

The main outcomes were critical care admission (high dependency unit or intensive care unit) and mortality in hospital or palliative discharge. We chose a priori to restrict analysis of outcomes to patients who were admitted more than two weeks before data extraction (3 May 2020) to enable most patients to finish their hospital admission.

\section{Bias}

Research nurses relied on local covid-19 test reports to enrol patients. Capacity to enrol was limited by staff resources at times of high covid-19 activity. Otherwise we are unable to comment on the potential selection bias of our cohort. We are in the process of linking to routine administrative healthcare data and will be able to make comparisons at that point.

\section{Missing data}

The nature of the study means that a large amount of data were missing, particularly during the later parts of the growth curve of the UK outbreak. Because this paper is mainly descriptive, we have not performed any imputation for missing data, and describe the data as they stand. To reduce the impact of missing data on outcome analyses, we restricted these analyses to patients who had been admitted for at least two weeks before data extraction.

\section{Statistical analyses}

Continuous data are summarised as median (interquartile range) and categorical data as frequency (percentage). For univariate comparisons, the MannWhitney U test or Kruskal-Wallis test were used. We compared categorical data by using the $\chi^{2}$ test.

We used several approaches to model survival. Discharge from hospital was considered an absorbing state, meaning that once discharged, patients were considered no longer at risk of death. Patients who were discharged were not censored and held within the risk set, therefore accounting for the competing risk of discharge on death. We checked this approach by using a formal Fine and Gray competing risks approach. Hierarchical Cox proportional hazards approaches included geographical region (clinical commissioning group or health board) as a random intercept. We used a parsimonious criterion based model building approach based on several principles: clinically relevant explanatory variables were identified a priori for exploration; population stratification was incorporated; interactions were checked at first order level; final model selection was informed by log likelihood tests and the concordance statistic, with appropriate assumptions checked including the distribution of residuals and requirement for proportional hazards. We set statistical significance at $5 \%$. All tests were two sided. We analysed data by using R (R Core Team version 3.6.3, Vienna, Austria), with packages including tidyverse, finalfit, survival, cmprsk, and coxme.

\section{Patient and public involvement}

This was an urgent public health research study in response to a Public Health Emergency of International Concern. Patients or the public were not involved in the design, conduct, or reporting of this rapid response research.

\section{Results}

On behalf of ISARIC WHO CCP-UK, 2468 research nurses, administrators, and medical students enrolled 20133 patients who were admitted with covid-19 to 208 hospitals in England, Scotland, and Wales between 6 February and 14:00 on 19 April 2020 (table 1 and fig E1). This figure represents 34\% of the 59215 covid-19 admissions in these countries. The median time from onset of symptoms of covid-19 in the community to presentation at hospital was 4 days (interquartile range $1-8 ; \mathrm{n}=16221$ ).

\section{Age and sex}

The median age of patients was 73 years (interquartile range 58-82, range 0-104; fig 1); 310 patients (1.5\%) were less than 18 years old and $194(1.0 \%)$ were less than 5 years old. More men $(59.9 \%, n=12068)$ than women $(40.1 \%, \mathrm{n}=8065)$ were admitted to hospital with covid-19. One hundred women (10\%) of reproductive age $(n=1033)$ were recorded as being pregnant.

\section{Symptoms}

The most common symptoms were cough $(68.9 \%$, $12896 / 18730)$, fever $(71.6 \%, 12499 / 17452)$, and shortness of breath $(71.2 \%, 12107 / 16999$; fig 2, top left panel), though these data reflect the case definition. Only 4.5\% (855/19178) of patients reported no symptoms on admission. We found a high degree of overlap between the three most common symptoms (fig 2, lower left panel).

Clusters of symptoms on admission were apparent (fig E2). The most common symptom cluster encompassed the respiratory system: cough, sputum, shortness of breath, and fever. We also observed three other clusters: one encompassing musculoskeletal symptoms (myalgia, joint pain, headache, and fatigue); a cluster of enteric symptoms (abdominal pain, vomiting, and diarrhoea); and less commonly, a mucocutaneous cluster. Twenty nine per cent (5384/18605) of all patients complained of enteric symptoms on admission, mostly in association with respiratory symptoms; however, $4 \%$ of all patients described enteric symptoms alone.

\section{Comorbidities}

Figure 2 (top right panel) and table 1 show major comorbidities recorded on admission. The most common major comorbidities were chronic cardiac disease (30.9\%, 5469/17 702), diabetes without complications (20.7\%, 3650/17599), chronic pulmonary disease excluding asthma $(17.7 \%, 3128 / 17634)$, chronic kidney disease $(16.2 \%, 2830 / 17506)$, and asthma 


\begin{tabular}{|c|c|c|c|}
\hline Characteristics & Male & Female & All \\
\hline Total No (\%) & $12068(59.9)$ & $8065(40.1)$ & 20133 \\
\hline \multicolumn{4}{|l|}{ Age at admission $(n=20133)$} \\
\hline Median (interquartile range) & $72.0(58.0-81.0)$ & $74.0(58.0-84.0)$ & $72.9(58.0-82.0)$ \\
\hline \multicolumn{4}{|l|}{ Age $(n=20133)$} \\
\hline$<18$ & $180(1.5)$ & $130(1.6)$ & $310(1.5)$ \\
\hline $18-39$ & $534(4.4)$ & $533(6.6)$ & $1067(5.3)$ \\
\hline $40-50$ & $888(7.4)$ & $530(6.6)$ & $1418(7.0)$ \\
\hline $50-59$ & $1728(14.3)$ & $980(12.2)$ & 2708 (13.5) \\
\hline $60-69$ & 2115 (17.5) & $1181(14.6)$ & $3296(16.4)$ \\
\hline $70-79$ & $2972(24.6)$ & $1720(21.3)$ & $4692(23.3)$ \\
\hline$\geq 80$ & $3651(30.3)$ & 2991 (37.1) & $6642(33.0)$ \\
\hline \multicolumn{4}{|l|}{ Any comorbidity $(n=18525)$} \\
\hline No & $2591(23.4)$ & $1570(21.1)$ & $4161(22.5)$ \\
\hline Yes & $8492(76.6)$ & $5872(78.9)$ & $14364(77.5)$ \\
\hline \multicolumn{4}{|c|}{ Chronic cardiac disease $(n=17702)$} \\
\hline No & $7086(66.8)$ & $5147(72.6)$ & $12233(69.1)$ \\
\hline Yes & $3527(33.2)$ & $1942(27.4)$ & $5469(30.9)$ \\
\hline \multicolumn{4}{|c|}{ Chronic pulmonary disease, not asthma $(n=17634)$} \\
\hline No & $8616(81.7)$ & $5890(83.1)$ & $14506(82.3)$ \\
\hline Yes & $1931(18.3)$ & $1197(16.9)$ & $3128(17.7)$ \\
\hline \multicolumn{4}{|l|}{ Asthma $(n=17535)$} \\
\hline No & $9274(88.6)$ & $5721(80.9)$ & $14995(85.5)$ \\
\hline Yes & $1192(11.4)$ & $1348(19.1)$ & $2540(14.5)$ \\
\hline \multicolumn{4}{|l|}{ Smoker $(n=14184)$} \\
\hline Never smoked & $5030(58.8)$ & $3938(69.9)$ & 8968 (63.2) \\
\hline Former smoker & $2972(34.8)$ & $1392(24.7)$ & $4364(30.8)$ \\
\hline Yes & $549(6.4)$ & $303(5.4)$ & $852(6.0)$ \\
\hline \multicolumn{4}{|c|}{ Chronic kidney disease $(\mathrm{n}=17506)$} \\
\hline No & $8792(84.0)$ & $5884(83.5)$ & $14676(83.8)$ \\
\hline Yes & $1671(16.0)$ & $1159(16.5)$ & $2830(16.2)$ \\
\hline \multicolumn{4}{|c|}{ Diabetes without complications ( $n=17599)$} \\
\hline No & $8254(78.3)$ & $5695(80.7)$ & $13949(79.3)$ \\
\hline Yes & $2290(21.7)$ & $1360(19.3)$ & $3650(20.7)$ \\
\hline \multicolumn{4}{|c|}{ Diabetes with complications $(\mathrm{n}=17516)$} \\
\hline No & $9628(91.8)$ & $6589(93.8)$ & $16217(92.6)$ \\
\hline Yes & $860(8.2)$ & $439(6.2)$ & $1299(7.4)$ \\
\hline \multicolumn{4}{|l|}{ Obesity $(n=16081)$} \\
\hline No & $8725(90.6)$ & $5671(87.8)$ & $14396(89.5)$ \\
\hline Yes & $900(9.4)$ & $785(12.2)$ & $1685(10.5)$ \\
\hline \multicolumn{4}{|c|}{ Chronic neurological disorder ( $n=17382)$} \\
\hline No & $9222(88.6)$ & $6189(88.7)$ & $15411(88.7)$ \\
\hline Yes & $1181(11.4)$ & $790(11.3)$ & $1971(11.3)$ \\
\hline \multicolumn{4}{|l|}{ Dementia $(n=17459)$} \\
\hline No & $9211(88.2)$ & $5888(83.9)$ & $15099(86.5)$ \\
\hline Yes & $1232(11.8)$ & $1128(16.1)$ & $2360(13.5)$ \\
\hline Malignancy $(n=17354)$ & & & \\
\hline No & $9251(89.2)$ & $6360(91.0)$ & $15611(90.0)$ \\
\hline Yes & $1117(10.8)$ & $626(9.0)$ & $1743(10.0)$ \\
\hline Moderate or severe liver disease & & & \\
\hline No & $10181(98.0)$ & $6869(98.5)$ & $17050(98.2)$ \\
\hline Yes & $204(2.0)$ & $106(1.5)$ & $310(1.8)$ \\
\hline Mild liver disease $(n=17331)$ & & & \\
\hline No & $10195(98.3)$ & $6855(98.5)$ & $17050(98.4)$ \\
\hline Yes & $174(1.7)$ & $107(1.5)$ & $281(1.6)$ \\
\hline Chronic haematological disease & & & \\
\hline No & $9951(96.0)$ & $6684(96.0)$ & $16635(96.0)$ \\
\hline Yes & $415(4.0)$ & $278(4.0)$ & $693(4.0)$ \\
\hline Rheumatological disorder $(\mathrm{n}=17$ & & & \\
\hline No & $9562(92.4)$ & $6031(86.9)$ & $15593(90.2)$ \\
\hline Yes & $787(7.6)$ & $909(13.1)$ & $1696(9.8)$ \\
\hline Malnutrition $(n=16695)$ & & & \\
\hline No & $9768(97.8)$ & $6531(97.4)$ & $16299(97.6)$ \\
\hline Yes & $222(2.2)$ & $174(2.6)$ & $396(2.4)$ \\
\hline Previous immunosuppressant $\mathrm{d}$ & $18009)$ & & \\
\hline Yes & $876(8.1)$ & $791(11.0)$ & $1667(9.3)$ \\
\hline No & $9339(86.6)$ & $6032(83.5)$ & $15371(85.4)$ \\
\hline Not applicable & $573(5.3)$ & $398(5.5)$ & $971(5.4)$ \\
\hline
\end{tabular}




\begin{tabular}{llll}
\hline $\begin{array}{l}\text { Table } 1 \mid \text { Continued } \\
\text { Characteristics }\end{array}$ & Male & Female & All \\
$\begin{array}{l}\text { Previous anti-infective treatment }(\mathrm{n}=18017) \\
\text { No }\end{array}$ & $1940(18.0)$ & $1311(18.2)$ & $3251(18.0)$ \\
\hline Yes & $8285(76.8)$ & $5520(76.4)$ & $13805(76.6)$ \\
\hline Not applicable & $569(5.3)$ & $392(5.4)$ & $961(5.3)$ \\
\hline AIDS/HIV $(\mathrm{n}=17$ 251) & & & $17168(99.5)$ \\
\hline No & $10259(99.5)$ & $6909(99.6)$ & $83(0.5)$ \\
\hline Yes & $55(0.5)$ & $28(0.4)$ & \\
\hline
\end{tabular}

(14.5\%, 2540/17535). Of 18525 patients, 22.5\% (4161) had no documented major comorbidity. There was little overlap between the three most common comorbidities (fig 2, lower right panel).

Six per cent $(852 / 14184)$ of patients were current smokers, 30.8\% (4364) were previous smokers, and
$63.2 \%$ (8968) had never smoked. Figure E3 shows the pattern of major comorbidity stratified by age.

\section{Level of care}

A high proportion of patients required admission to high dependency or intensive care units $(17 \%$,
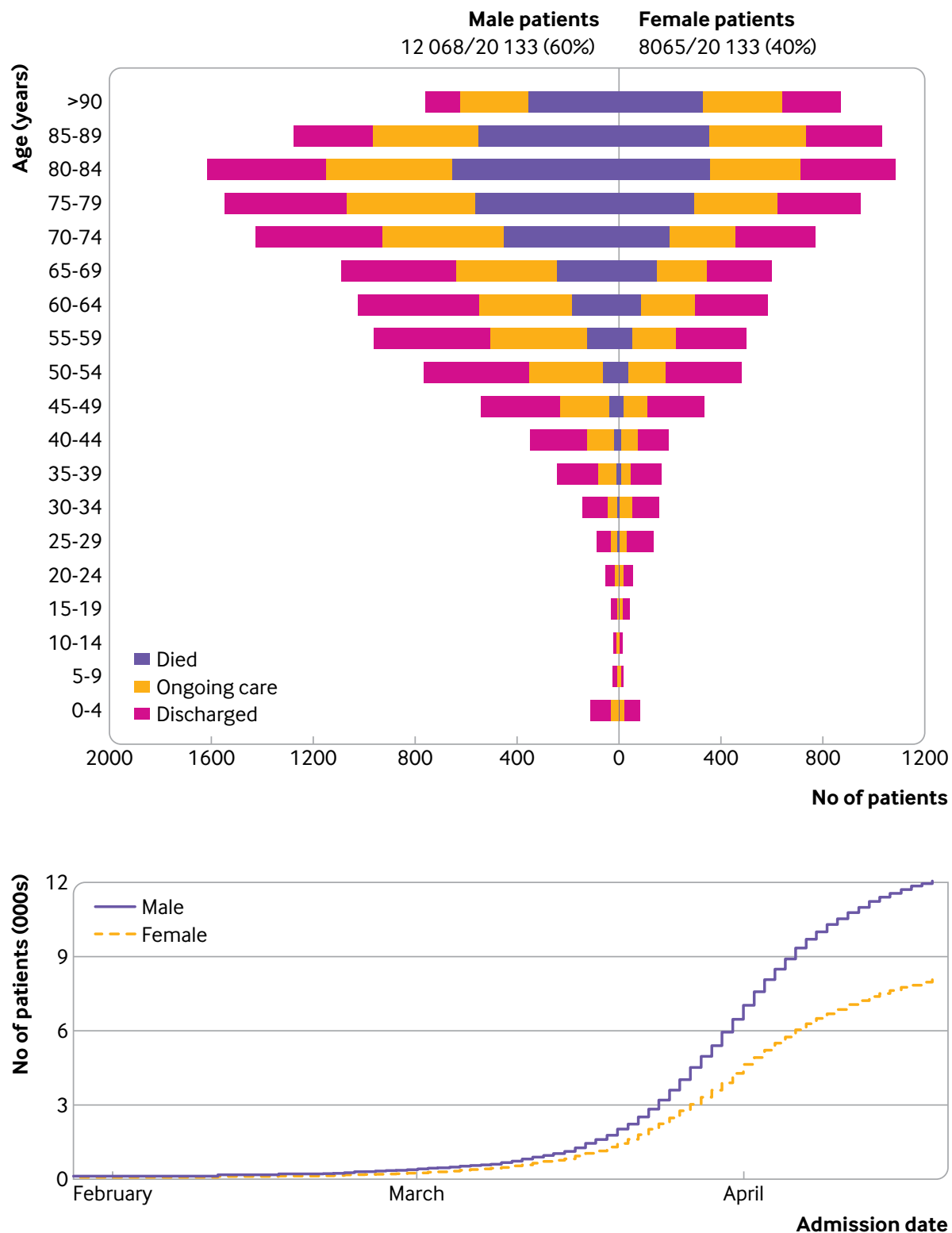

Fig 1 | Patients with coronavirus disease 2019 (covid-19) stratified by age and sex (top panel), and date of hospital admission with covid-19 by sex (lower panel). Outcomes are discharge from hospital, ongoing care, and death at time of report (19 April 2020, $n=20133$ ) 
3001/18 183; fig 3), and 55\% (9244/16 849) received high flow oxygen at some point during their admission. Sixteen per cent of patients (2670/16805) were treated with non-invasive ventilation, while $10 \%$ (1658/16 866) received invasive ventilation.

\section{Patient outcomes}

Overall, 41\% (8199/20133) of patients were discharged alive, 26\% (5165/20133) died, and 34\% $(6769 / 20133)$ continued to receive care at the date of reporting (fig 4). The median age of patients who died
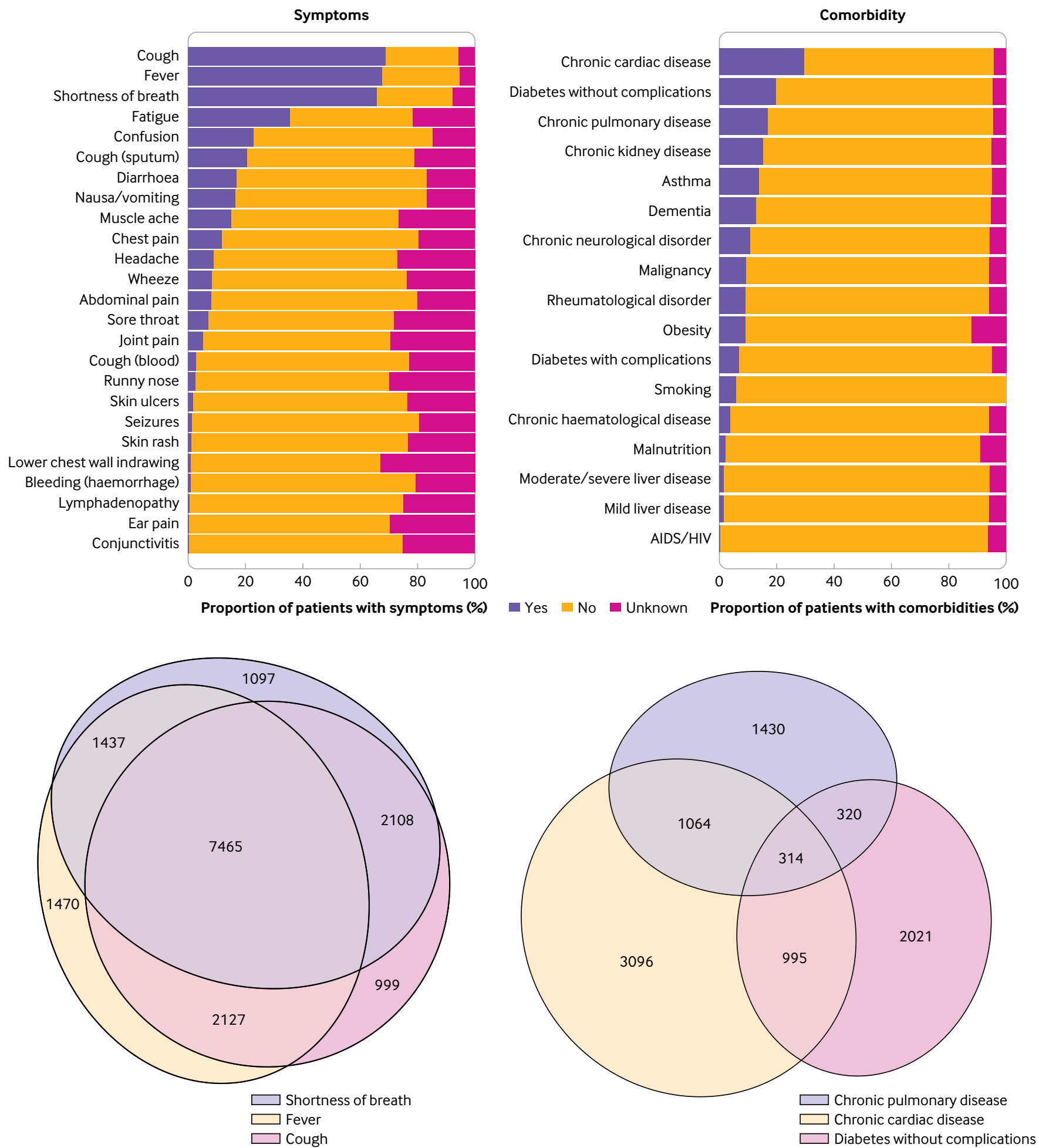

Fig 2 | Presenting symptoms and comorbidities in patients in hospital with coronavirus disease 2019 (covid-19). Top left panel: symptoms by frequency of presentation (see table E1 for values); lower left panel: scaled Euler diagram of overlap of commonest symptoms; top right panel: comorbidities by frequency (see table 1 for values); lower right panel: scaled Euler diagram of overlap of commonest comorbidities 
in hospital from covid-19 in the study was 80 years, and only $11 \%(559 / 4880)$ of these patients had no documented major comorbidity.

For patients who received only ward care, $47 \%(7203 / 15297)$ were discharged alive, 26\% (3954/15 297) died, and 27\% (4140/15 297) remained in hospital at the date of reporting. As expected, outcomes were worse for those who needed higher levels of care.

Of patients admitted to critical care (high dependency unit or intensive care unit), 28\% (826/3001) were discharged alive, 32\% (958/3001) died, and $41 \%(1217 / 3001)$ continued to receive care at the date of reporting. Although the patients who received mechanical ventilation were younger than the overall cohort (61 years, interquartile range 52-69), only $17 \%$ (276/1658) had been discharged alive by 19 April 2020, 37\% (618/1658) had died, and 46\% (764/1658) continued to receive care.

Length of stay increased with age for patients discharged alive (fig E4). For patients who died, we found no association between age and time to death, with around $80 \%$ dying before day 14 of hospital admission.

\section{Association of pre-existing patient characteristics and survival}

The online supplement (table E4) describes univariable and multivariable associations with mortality. Figure 5 shows variables that remained significant in the multivariable model. Increasing age was a strong predictor of mortality in hospital after adjusting for major comorbidity (reference age $<50$ years): 50-59 years, hazard ratio 2.63 (95\% confidence interval 2.06 to 3.35, $\mathrm{P}<0.001$ ); 60-69 years, 4.99 (3.99 to 6.25, $\mathrm{P}<0.001$ ); 70-79 years, 8.51 (6.85 to $10.57, \mathrm{P}<0.001$ ); $\geq 80$ years, 11.09 (8.93 to 13.77, $\mathrm{P}<0.001$ ). Female sex was associated with lower mortality $(0.81,0.75$ to $0.86, \mathrm{P}<0.001)$. Chronic cardiac disease, chronic non-asthmatic pulmonary disease, chronic kidney disease, obesity, chronic neurological disorder (such as stroke), dementia, malignancy, and liver disease were also associated with increased hospital mortality. An interactive infographic is available at https:// isaric4c.net/info. This information must not be used as a predictive tool in practice or to inform individual treatment decisions.

\section{Discussion}

\section{Principal findings}

Patients with covid-19 usually presented with fever, cough, and shortness of breath, and met the WHO case definitions for severe acute respiratory infection or severe acute respiratory syndrome. The most common previous major comorbidities were chronic cardiac disease, diabetes, and chronic non-asthmatic pulmonary disease. Seventeen per cent of patients were admitted to critical care (high dependency unit or intensive care unit). Mortality in hospital was at least $26 \%$, with $34 \%$ of the cohort still in hospital at the time of analysis; these proportions increased with escalating level of care. Factors associated with mortality in hospital were increasing age, male sex, and major comorbidities (cardiac disease, non-
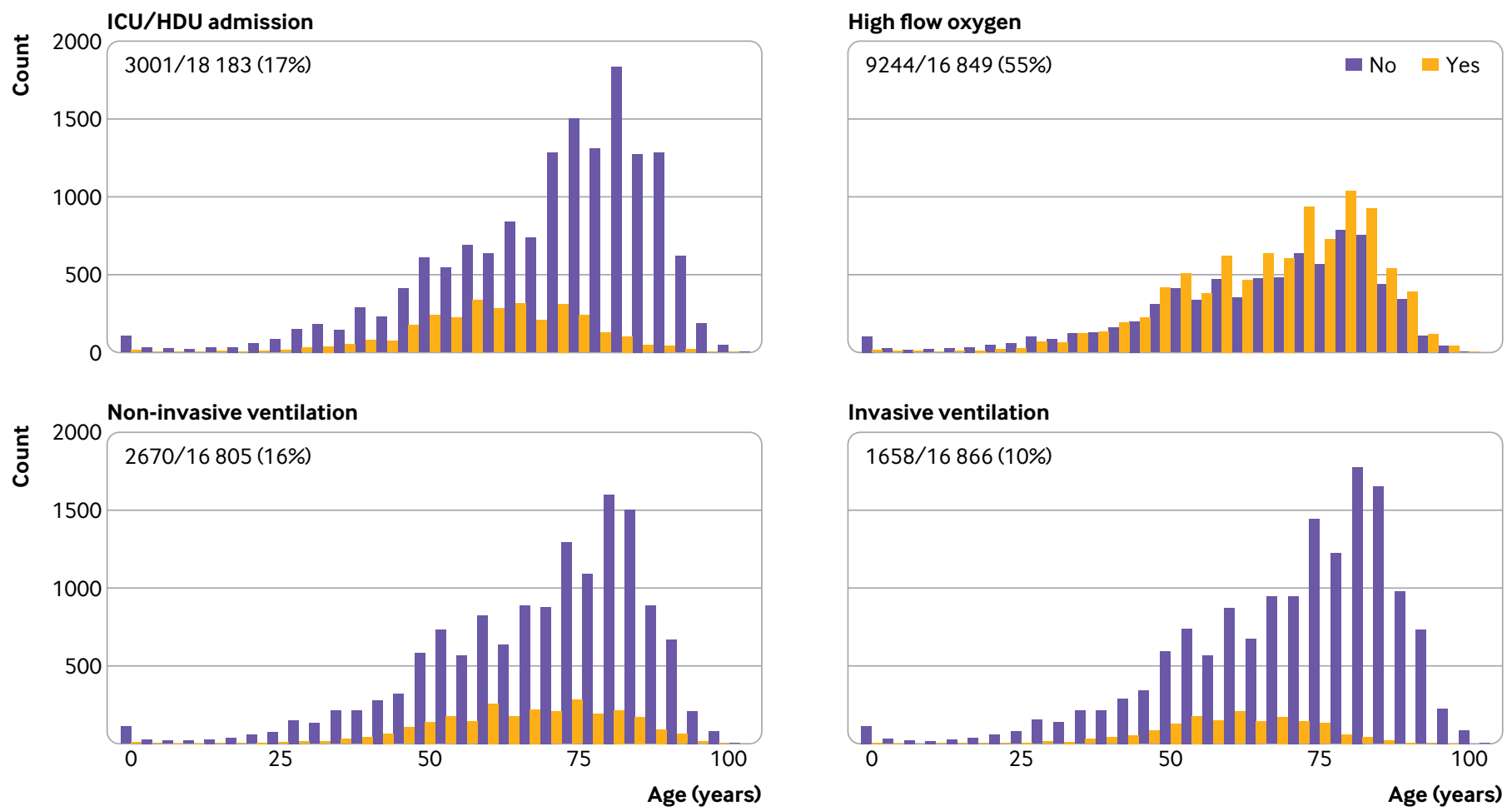

Fig 3 | Level of care stratified by age: admitted to intensive care unit (ICU) or high dependency unit (HDU), high flow oxygen, non-invasive ventilation, and invasive ventilation 
Died
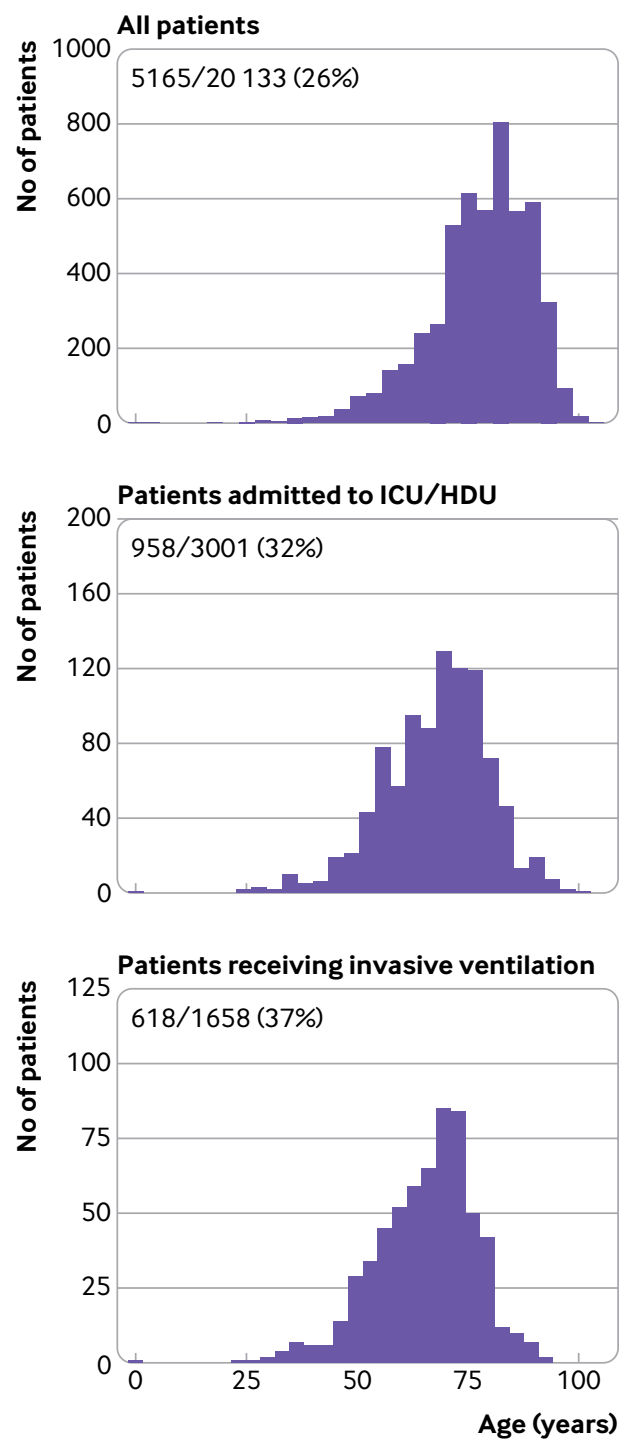

Ongoing care
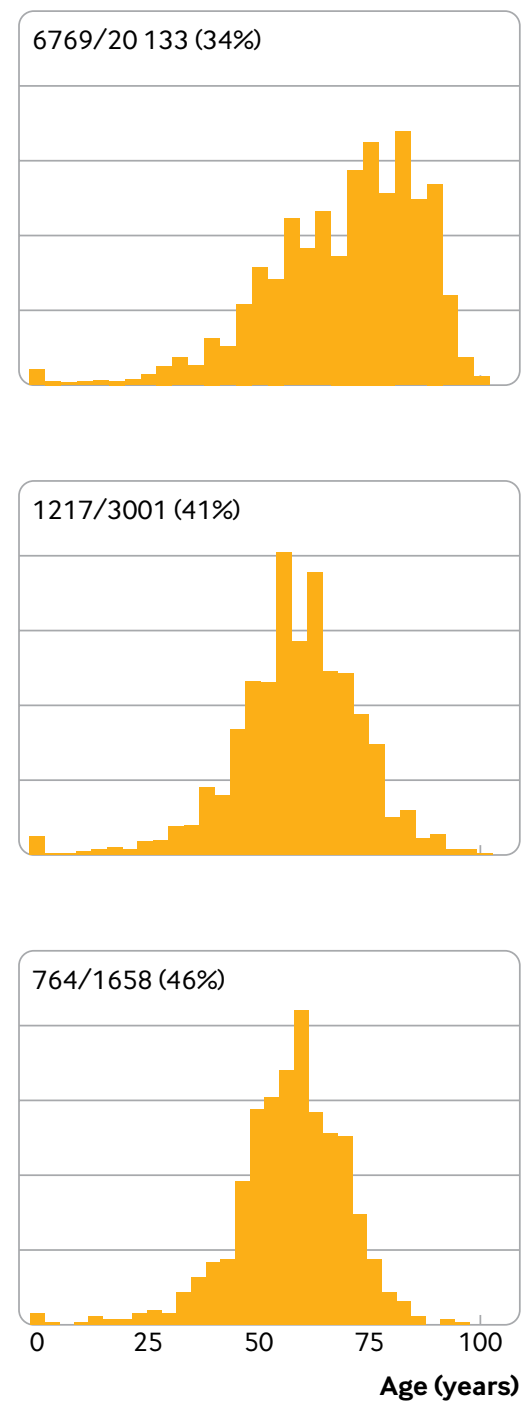

Discharged
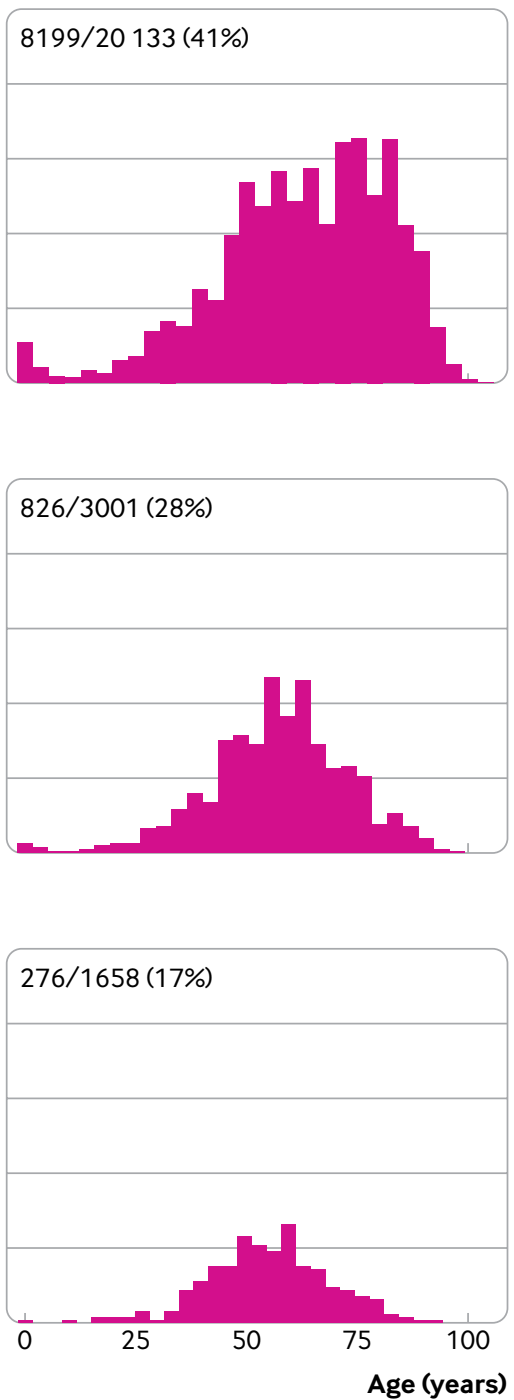

Fig 4 | Status of patients at time of reporting stratified by level of care. Top panel: all patients in hospital with coronavirus disease 2019 (covid-19); middle panel: all patients admitted to intensive care unit (ICU) or high dependency unit (HDU); lower panel: patients receiving invasive mechanical ventilation

asthmatic pulmonary disease, kidney disease, liver disease, malignancy, obesity, and dementia).

The data presented in this study describe patients admitted to hospital during the growth phase of the SARS-CoV-2 pandemic in the UK. The first 101 patients were enrolled in the early phase of the outbreak as part of a high consequence infectious disease containment strategy that ended on 10 March 2020. These patients and others who were identified through screening in hospital, or who contracted covid-19 after admission (hospital acquired infection), are included in the 855 patients who were admitted without covid-19 symptoms. The impact these patients have had on the overall cohort characteristics has diminished as numbers have increased, and we believe it is important to keep these patients in the study. Other patients in our cohort without covid-19 symptoms are those who were diagnosed with the disease at the discretion of the clinician looking after them while staying in hospital for other reasons.

The pattern of disease we describe broadly reflects the pattern reported globally. ${ }^{7}$ Patients in our study had a higher median age and higher rates of chronic obstructive pulmonary disease and asthma than patients in China ${ }^{8}$ and the US. ${ }^{11} 12$ The prevalence of obesity in our study (11\%) was considerably lower than the overall UK prevalence (29\%). ${ }^{13}$ This proportion could reflect the relatively elderly male population admitted to hospital and misclassification or under reporting by admitting physicians. Our patients presented with a relatively short time interval between onset of symptoms and admission to hospital, which might also be a function of the older and vulnerable patient population.

The current case definition of cough and fever, if strictly applied, would miss $7 \%$ of our inpatients. 


\begin{tabular}{|c|c|c|c|c|c|c|c|}
\hline & & \multicolumn{4}{|c|}{$\begin{array}{l}\text { Hazard ratio } \\
(95 \% \mathrm{Cl})\end{array}$} & $\begin{array}{l}\text { Hazard ratio } \\
\quad(95 \% \mathrm{Cl})\end{array}$ & $\begin{array}{c}P \\
\text { value }\end{array}$ \\
\hline \multirow[t]{5}{*}{ Age on admission (years) } & $<50$ & . & & & & & \\
\hline & $50-59$ & & & & & $2.63(2.06$ to 3.35$)$ & $<0.001$ \\
\hline & $60-69$ & & & - & & 4.99 (3.99 to 6.25$)$ & $<0.001$ \\
\hline & $70-79$ & & & & $\longrightarrow$ & $8.51(6.85$ to 10.57$)$ & $<0.001$ \\
\hline & $\geq 80$ & & & & $\longrightarrow$ & 11.09 (8.93 to 13.77$)$ & $<0.001$ \\
\hline Sex at birth & Female & $\bullet$ & & & & $0.81(0.75$ to 0.86$)$ & $<0.001$ \\
\hline Chronic cardiac disease & Yes & & $\leftrightarrow$ & & & $1.16(1.08$ to 1.24$)$ & $<0.001$ \\
\hline Chronic pulmonary disease & Yes & & $\leftrightarrow-$ & & & $1.17(1.09$ to 1.27$)$ & $<0.001$ \\
\hline Chronic kidney disease & Yes & & $\rightarrow$ & & & 1.28 (1.18 to 1.39$)$ & $<0.001$ \\
\hline Diabetes & Yes & & 4 & & & $1.06(0.99$ to 1.14$)$ & 0.087 \\
\hline Obesity & Yes & & -•- & & & 1.33 (1.19 to 1.49$)$ & $<0.001$ \\
\hline Chronic neurological disorder & Yes & & $\rightarrow$ & & & $1.17(1.06$ to 1.29$)$ & 0.001 \\
\hline Dementia & Yes & & $-\infty$ & & & 1.40 (1.28 to 1.52$)$ & $<0.001$ \\
\hline Malignancy & Yes & & $-\bullet$ & & & $1.13(1.02$ to 1.24$)$ & 0.017 \\
\hline \multirow[t]{2}{*}{ Moderate/severe liver disease } & e Yes & & - - & & & $1.51(1.21$ to 1.88$)$ & $<0.001$ \\
\hline & & 1 & 2 & 5 & 10 & & \\
\hline
\end{tabular}

Fig 5 | Multivariable Cox proportional hazards model (age, sex, and major comorbidities), where hazard is death. Patients who were discharged were kept in the risk set $(n=15194$; No of events=3911)

A smaller proportion, $4 \%$ of patients, presented with enteric symptoms only. This figure could be an underestimate because these patients fall outside standard criteria for testing. This enteric presentation risks misclassification of patients, and assignment to non-covid-19 care areas, which could pose a nosocomial transmission risk. Severe SARS-CoV-2 infections are rare in people younger than 18 years, comprising only $1.5 \%$ of those admitted to hospital. Only $1.0 \%$ of those in our study were younger than 5 years. The J shaped age distribution is starkly different to the $U$ shaped age distribution seen in seasonal influenza and the $\mathrm{W}$ shaped distribution observed in the 2009 influenza pandemic. ${ }^{14}$ The reason why SARSCoV-19 has mostly spared children is not clear, but we speculate this could be because angiotensin converting enzyme 2 receptors are expressed differently in younger lungs.

Other studies have not widely reported that obesity as recognised by clinical staff is associated with mortality in hospital after adjustment for other comorbidities, age, and sex. Obesity was recognised as a risk factor in the 2009 influenza A H1N1 pandemic, but not for the 2012 Middle East respiratory syndrome coronavirus. ${ }^{1516}$

The proportion of pregnant women in our cohort was small $(10 \%)$, similar to the estimated proportion of pregnant women in the community. ${ }^{15}$ Pregnancy was not associated with mortality, in apparent contrast to influenza. ${ }^{17}$

\section{Comparison with other studies}

The proportion of patients admitted to critical care in our study was similar to that reported in Italy $(17 \%),{ }^{18}{ }^{19}$ and New York (14.2\%), ${ }^{11}{ }^{12}$ but higher than China. ${ }^{8}$ At the time of enrolment, the Intensive
Care Society had issued guidance to its members that there would be no rationing of critical care admission until all capacity in the country had been exhausted. As far as we are aware, critical care capacity was not exceeded in the UK during the period of the study. We do not believe that any equipment shortages existed during this period that might have prompted more aggressive futility discussions.

Mortality in our cohort was high in patients admitted to general wards who were not admitted to critical care, which suggests that advanced care planning occurred. We were unable to capture treatment limiting decisions about level of care. The high median age of patients who died in the cohort (80 years) could partly explain the high mortality rate. Mortality rates were extremely high for patients who received invasive mechanical ventilation in the intensive care unit compared with the 2009 influenza A H1N1 pandemic, for which mortality in intensive care was $31 \% .{ }^{15}$ Our data were in line with the initial ICNARC (intensive care national audit and research centre) audit reports, which represent intensive care units in England, Wales, Scotland, and Northern Ireland. ${ }^{20}$

Outcome analyses only included patients who were admitted before 19 April to allow most patients to complete their hospital admission. However, an inherent reporting bias exists because the sickest of patients, particularly those admitted to intensive care, have the longest hospital stays; mortality rates in hospital could therefore increase. These mortality rates were considerably higher than the $24 \%$ mortality rate in hospital seen in patients in intensive care units in Italy ${ }^{19}$ and the US. ${ }^{11} 12$ The lower rate in the US could in part be explained by differences in healthcare systems and the proportion of intensive care unit beds to hospital beds between the two countries. In Italy, 
a lower proportion of patients received mechanical ventilation, and most of their patients (72\%) remained in hospital at the time of the analysis. ${ }^{19}$

The finding of independent associations of advancing age, male sex, chronic respiratory (nonasthmatic) disease, chronic cardiac disease, and chronic neurological disease with mortality in hospital is in line with early international reports. ${ }^{910}$ However, although age adjusted mortality rates are high in elderly patients, most of these patients were admitted to hospital with symptoms of covid-19 and would not have been in hospital otherwise. Enhanced severity in male patients was seen across all ages.

\section{Strengths and limitations of study}

ISARIC WHO CCP-UK stood ready to conduct large scale studies of pandemic outbreaks for eight years, enabling us to enrol $34 \%$ of all patients with covid-19 admitted to 208 acute care hospitals across England, Wales and Scotland in the early phase of the pandemic.

Our study has some limitations. We do not currently have data on the inpatients that were not enrolled, or people managed in community settings, such as usual domestic residences and older people's care homes. We are unable to comment on community risk factors that drive hospital admission except by inference from expected representation at admission. We will be linking to routine administrative healthcare datasets which will enable us to assess the presence of any selection bias.

A large amount of data were missing and we suggest there are two main reasons for this. Firstly, enrolment occurred in the nonlinear growth phase of the outbreak, and outcomes for recent admissions have not been reported yet; these admissions account for $18 \%$ of the total number of patients enrolled. Secondly, the research network was dealing with unprecedented numbers of patients at a time when many were seconded to clinical practice or themselves off sick. This study is ongoing, and further data are being added to case report forms.

We suggest it is possible that the sickest patients were enrolled in our study, and this could partly explain our high mortality rates in hospital. Some of the sickest patients in the study had the longest lengths of hospital stay and we do not have outcome data for all of these patients yet.

\section{Conclusions and policy implications}

This large and rapidly conducted study of patients admitted to hospital in England, Wales, and Scotland with covid-19 shows the importance of putting plans in place for the study of epidemic and pandemic threats, and the need to maintain these plans. Our study identifies sectors of the population that are at greatest risk of a poor outcome, and reports the use of healthcare resources. Most patients with covid-19 experience mild disease. However, in our cohort, of those who were admitted to hospital two weeks before data extraction, less than half have been discharged alive and a quarter have died. The remainder continued to receive care at the date of reporting. Seventeen percent of patients admitted to hospital required critical care. Factors associated with mortality in hospital were increasing age, male sex, obesity, and major comorbidities.

ISARIC Coronavirus Clinical Characterisation Consortium $^{21}$ investigators have submitted regular reports to the UK Government's New and Emerging Respiratory Virus Threats Advisory Group (NERVTAG) ${ }^{22}$ and the Scientific Advisory Group for Emergencies (SAGE). ${ }^{23}$ Patient level data have been shared and independently analysed by the Scientific Pandemic Influenza Group on Modelling (SPI-M) ${ }^{24}$ and other investigators. Aggregated data have been shared with WHO in the ISARIC covid-19 report.

Studies such as this cannot be developed, approved, and opened from the start of a pandemic in time to inform case management and public health policy. Our study has shown the importance of forward planning and investment in preparedness studies. Over the next few months we will issue reports in The BMJ on specific topics and analyses that are key to understanding the impact of covid-19 and focus on improving patient outcomes.

\section{AUTHOR AFFILIATIONS}

${ }^{1}$ Centre for Medical Informatics, Usher Institute, University of Edinburgh, Edinburgh, UK

${ }^{2}$ Intensive Care Unit, Royal Infirmary Edinburgh, Edinburgh, UK ${ }^{3}$ Institute of Microbiology and Infection, University of Birmingham, Birmingham, UK

${ }^{4}$ National Institute of Health Research (NIHR) Health Protection Research Unit in Emerging and Zoonotic Infections, Liverpool, UK

${ }^{5}$ Institute of Infection and Global Health, Faculty of Health and Life Sciences, University of Liverpool, Liverpool, UK

${ }^{6}$ Institute of Translational Medicine, Faculty of Health and Life Sciences, University of Liverpool, Liverpool, UK

${ }^{7}$ Centre for Health Informatics, Computing and Statistics, Lancaster Medical School, Lancaster University, Bailrigg, UK

${ }^{8}$ ISARIC Global Support Centre, Centre for Tropical Medicine and Global Health, Nuffield Department of Medicine, University of Oxford, Oxford, UK

${ }^{9}$ Infectious Diseases Data Observatory, Centre for Tropical Medicine and Global Health, University of Oxford, Oxford, UK

${ }^{10}$ Liverpool Clinical Trials Centre, University of Liverpool, Liverpool, UK

${ }^{11}$ Centre for Tropical Medicine and International Health, Nuffield Department of Medicine, University of Oxford, Oxford, UK

${ }^{12}$ Division of Epidemiology and Public Health, University of Nottingham School of Medicine, Nottingham, UK

${ }^{13}$ Medical Research Council University of Glasgow Centre for Virus Research, Glasgow, UK

${ }^{14}$ Queen's Medical Research Institute, University of Edinburgh, Edinburgh, UK

${ }^{15}$ National Infection Service, Public Health England, London, UK

${ }^{16}$ Faculty of Medicine, Imperial College London, London, UK

${ }^{17}$ National Heart and Lung Institute, Faculty of Medicine, Imperial College London, London, UK

${ }^{18}$ Roslin Institute, University of Edinburgh, Edinburgh, UK

${ }^{19}$ NIHR Health Protection Research Unit in Emerging and Zoonotic Infections and Institute of Translational Medicine, Faculty of Health and Life Sciences, University of Liverpool, Liverpool, UK

${ }^{20}$ Respiratory Medicine, Alder Hey Children's Hospital, Institute in The Park, University of Liverpool, Alder Hey Children's Hospital, Liverpool L12 2AP, UK

The study protocol is available at http://isaric4c.net/protocols; study registry https://www.isrctn.com/ISRCTN66726260. This work uses data provided by patients and collected by the NHS as part of their 
care and support \#DataSavesLives. We are extremely grateful to the 2648 frontline NHS clinical and research staff and volunteer medical students who collected these data in challenging circumstances; and the generosity of the patients and their families for their individual contributions in these difficult times. We also acknowledge the support of Jeremy J Farrar, Nahoko Shindo, Devika Dixit, Nipunie Rajapakse, Piero Olliaro, Lyndsey Castle, Martha Buckley, Debbie Malden, Katherine Newell, Kwame O’Neill, Emmanuelle Denis, Claire Petersen, Scott Mullaney, Sue MacFarlane, Chris Jones, Nicole Maziere, Katie Bullock, Emily Cass, William Reynolds, Milton Ashworth, Ben Catterall, Louise Cooper, Terry Foster, Paul Matthew Ridley, Anthony Evans, Catherine Hartley, Chris Dunn, D Sales, Diane Latawiec, Erwan Trochu, Eve Wilcock, Innocent Gerald Asiimwe, Isabel Garcia-Dorival, J Eunice Zhang, Jack Pilgrim, Jane A Armstrong, Jordan J Clark, Jordan Thomas, Katharine King, Katie Neville, Alexandra Ahmed, Krishanthi S Subramaniam, Lauren Lett, Laurence McEvoy, Libby van Tonder, Lucia Alicia Livoti, Nahida S Miah, Rebecca K Shears, Rebecca Louise Jensen, Rebekah Penrice-Randal, Robyn Kiy, Samantha Leanne Barlow, Shadia Khandaker, Soeren Metelmann, Tessa Prince, Trevor R Jones, Benjamin Brennan, Agnieska Szemiel, Siddharth Bakshi, Daniella Lefteri, Maria Mancini, Julien Martinez, Angela Elliott, Joyce Mitchell, John McLauchlan, Aislynn Taggart, Oslem Dincarslan, Annette Lake, Claire Petersen, Scott Mullaney, and Graham Cooke.

ISARIC Coronavirus Clinical Characterisation Consortium (ISARIC4C) Consortium lead investigator: I Kenneth Baillie; chief investigator: Malcolm G Semple; co-lead investigator: Peter JM Openshaw; ISARIC clinical coordinator: Gail Carson; co-investigators: Beatrice Alex, Benjamin Bach, Wendy S Barclay, Debby Bogaert, Meera Chand, Graham S Cooke, Annemarie B Docherty, Jake Dunning, Ana da Silva Filipe, Tom Fletcher, Christopher A Green, Julian A Hiscox, Antonia Ying Wai Ho, Peter W Horby, Samreen ljaz, Saye Khoo, Paul Klenerman, Andrew Law, Wei Shen Lim, Alexander J Mentzer, Laura Merson, Alison M Meynert, Mahdad Noursadeghi, Shona C Moore, Massimo Palmarini, William A Paxton, Georgios Pollakis, Nicholas Price, Andrew Rambaut, David L Robertson, Clark D Russell, Vanessa Sancho-Shimizu, Janet T Scott, Tom Solomon, Shiranee Sriskandan, David Stuart, Charlotte Summers, Richard S Tedder, Emma C Thomson, Ryan S Thwaites, Lance CW Turtle, Maria Zambon; project managers: Hayley E Hardwick, Chloe Donohue, Jane Ewins, Wilna Oosthuyzen, Fiona Griffiths; data analysts: Lisa Norman, Riinu Pius, Tom M Drake, Cameron J Fairfield, Stephen Knight, Kenneth A Mclean, Derek Murphy, Catherine A Shaw; data and information system managers: Jo Dalton, Michelle Girvan, Egle Saviciute, Stephanie Roberts, Janet Harrison, Laura Marsh, Marie Connor; data integration and presentation: Gary Leeming, Andrew Law, Ross Hendry; material management: William Greenhalf, Victoria Shaw, Sarah McDonald; local principal investigators: Kayode Adeniji, Daniel Agranoff, Ken Agwuh, Dhiraj Ail, Ana Alegria, Brian Angus, Abdul Ashish, Dougal Atkinson, Shahedal Bari, Gavin Barlow, Stella Barnass, Nicholas Barrett, Christopher Bassford, David Baxter, Michael Beadsworth, Jolanta Bernatoniene, John Berridge, Nicola Best, Pieter Bothma, David Brealey, Robin Brittain-Long, Naomi Bulteel, Tom Burden, Andrew Burtenshaw, Vikki Caruth, David Chadwick, Duncan Chambler, Nigel Chee, Jenny Child, Srikanth Chukkambotla, Tom Clark, Paul Collini, Graham Cooke, Catherine Cosgrove, Jason Cupitt, Maria-Teresa Cutino-Moguel, Paul Dark, Chris Dawson, Samir Dervisevic, Phil Donnison, Sam Douthwaite, Ingrid DuRand, Ahilanadan Dushianthan, Tristan Dyer, Cariad Evans, Chi Eziefula, Chrisopher Fegan, Adam Finn, Duncan Fullerton, Sanjeev Garg, Sanjeev Garg, Atul Garg, Effrossyni Gkrania-Klotsas, Jo Godden, Arthur Goldsmith, Clive Graham, Elaine Hardy, Stuart Hartshorn, Daniel Harvey, Peter Havalda, Daniel B Hawcutt, Antonia Ho, Maria Hobrok, Luke Hodgson, Anita Holme, Anil Hormis, Michael Jacobs, Susan Jain, Paul Jennings, Agilan Kaliappan, Vidya Kasipandian, Stephen Kegg, Michael Kelsey, Jason Kendall, Caroline Kerrison, Ian Kerslake, Oliver Koch, Gouri Koduri, George Koshy, Shondipon Laha, Susan Larkin, Tamas Leiner, Patrick Lillie, James Limb, Vanessa Linnett, Jeff Little, Michael MacMahon, Emily MacNaughton, Ravish Mankregod, Huw Masson, Elijah Matovu, Katherine McCullough, Ruth McEwen, Manjula Meda, Gary Mills, Jane Minton, Mariyam Mirfenderesky, Kavya Mohandas, James Moon, Elinoor Moore, Patrick Morgan, Craig Morris, Katherine Mortimore, Samuel Moses, Mbiye Mpenge, Rohinton Mulla, Michael Murphy, Megan Nagel, Thapas Nagarajan, Mark Nelson, Igor Otahal, Mark Pais, Selva Panchatsharam, Hassan Paraiso, Brij Patel, Justin Pepperell, Mark Peters, Mandeep Phull, Stefania Pintus, Jagtur Singh Pooni, Frank Post, David Price, Rachel Prout, Nikolas Rae, Henrik Reschreiter, Tim Reynolds, Neil Richardson, Mark Roberts, Devender Roberts, Alistair Rose, Guy Rousseau, Brendan Ryan, Taranprit Saluja, Aarti Shah, Prad Shanmuga, Anil Sharma, Anna Shawcross, Jeremy Sizer, Richard Smith, Catherine Snelson, Nick Spittle, Nikki Staines, Tom Stambach, Richard Stewart, Pradeep Subudhi, Tamas Szakmany,
Kate Tatham, Jo Thomas, Chris Thompson, Robert Thompson, Ascanio Tridente, Darell Tupper-Carey, Mary Twagira, Andrew Ustianowski, Nick Vallotton, Lisa Vincent-Smith, Shico Visuvanathan, Alan Vuylsteke, Sam Waddy, Rachel Wake, Andrew Walden, Tony Whitehouse, Paul Whittaker, Ashley Whittington, Meme Wijesinghe, Martin Williams, Lawrence Wilson, Sarah Wilson, Stephen Winchester, Martin Wiselka, Adam Wolverson, Daniel G Wooton, Andrew Workman, Bryan Yates, Peter Young.

Contributors: Conceptualisation: JKB, JD, GC, LM, JSN-V-T, PJMO, MGS. Formal analysis: ABD, FD, CG, EMH, PWH, LN, PJMO, RP, JMR, MGS Writing original draft: ABD, PJMO, MGS. Writing reviewing and editing: IKB, ABD, JD, CG, CAG, EMH, PWH, JSN-V-T, PJMO, MGS, LS. Project administration: $\mathrm{SH}, \mathrm{HEH}, \mathrm{CG}, \mathrm{AH}, \mathrm{KAH}$, JL, LM, DP, CDR. Investigation: EMH, PWH, CG, CAG, AH, MGS. Supervision: JKB, HEH, EMH, CG, AH, PWH, PJMO, MGS. Data curation: LM, SH, CJ. Validation: KAH, SH, CJ. Funding acquisition: JKB, GC, PWH, PJMO, MGS. MGS is guarantor and corresponding author for this work, and attests that all listed authors meet authorship criteria and that no others meeting the criteria have been omitted.

Funding: This work is supported by grants from: the National Institute for Health Research (NIHR; award CO-CIN-01), the Medical Research Council (MRC; grant MC_PC_19059), the NIHR Health Protection Research Unit in Emerging and Zoonotic Infections at University of Liverpool in partnership with Public Health England (PHE), in collaboration with Liverpool School of Tropical Medicine and the University of Oxford (NIHR award 200907), Wellcome Trust and Department for International Development (DID; 215091/Z/18/Z), and the Bill and Melinda Gates Foundation (OPP1209135), and Liverpool Experimental Cancer Medicine Centre for providing infrastructure support for this research (grant reference C18616/ A25153). JSN-V-T is seconded to the Department of Health and Social Care, England (DHSC). The views expressed are those of the authors and not necessarily those of the DHSC, DID, NIHR, MRC, Wellcome Trust, or PHE.

Competing interests: All authors have completed the ICMJE uniform disclosure form at www.icmje.org/coi_disclosure.pdf and declare: support from the National Institute for Health Research (NIHR), the Medical Research Council (MRC), the NIHR Health Protection Research Unit (HPRU) in Emerging and Zoonotic Infections at University of Liverpool, and Public Health England (PHE), Wellcome Trust, Department for International Development (DID), the Bill and Melinda Gates Foundation, Liverpool Experimental Cancer Medicine Centre, and Department of Health and Social Care (DHSC) for the submitted work; ABD reports grants from DHSC during the conduct of the study; grants from Wellcome Trust outside the submitted work; CAG reports grants from DHSC NIHR UK during the conduct of the study; FD is due to start a position at F Hoffmann-La Roche on 4 May 2020; PWH reports grants from Wellcome Trust, DID, Bill and Melinda Gates Foundation, and NIHR during the conduct of the study; JSN-V-T reports grants from DHSC during the conduct of the study, and is seconded to DHSC; PJMO reports personal fees from consultancies and from the European Respiratory Society; grants from MRC, MRC Global Challenge Research Fund, EU, NIHR Biomedical Research Centre, MRC/GSK, Wellcome Trust, NIHR (HPRU in Respiratory Infection), and is an NIHR senior investigator outside the submitted work; his role as President of the British Society for Immunology was unpaid but travel and accommodation at some meetings was provided by the Society; JKB reports grants from MRC UK; MGS reports grants from DHSC NIHR UK, MRC UK, HPRU in Emerging and Zoonotic Infections, University of Liverpool during the conduct of the study; other from Integrum Scientific LLC, Greensboro, NC, US outside the submitted work; the remaining authors declare no competing interests; no financial relationships with any organisations that might have an interest in the submitted work in the previous three years; and no other relationships or activities that could appear to have influenced the submitted work.

Ethical approval: Ethical approval was given by the South Central Oxford C Research Ethics Committee in England (Ref 13/SC/0149), the Scotland A Research Ethics Committee (Ref 20/SS/0028), and the WHO Ethics Review Committee (RPC571 and RPC572, 25 April 2013).

Data sharing: We welcome applications for data and material access through our Independent Data and Material Access Committee (https://isaric4c.net).

The lead author (the manuscript's guarantor) affirms that the manuscript is an honest, accurate, and transparent account of the study being reported; that no important aspects of the study have been omitted; and that any discrepancies from the study as planned (and, if relevant, registered) have been explained. 
Dissemination to participants and related patient and public communities: ISARIC4C has a public facing website and twitter account @CCPUKstudy. We are engaging with print and internet press, television, radio, news, and documentary programme makers. We will explore distribution of findings with The Asthma UK and British Lung Foundation Partnership, and take advice from NIHR Involve and GenerationR Alliance Young People’s Advisory Groups.

This is an Open Access article distributed in accordance with the terms of the Creative Commons Attribution (CC BY 4.0) license, which permits others to distribute, remix, adapt and build upon this work, for commercial use, provided the original work is properly cited. See: http://creativecommons.org/licenses/by/4.0/.

1 WHO. WHO Director-General's opening remarks at the media briefing on COVID-19 - 11 March 2020 https.//www who.int/dg/speeches/ detail/who-director-general-s-opening-remarks-at-the-mediabriefing-on-covid-19---11-march-2020.

2 WHO WHO coronavirus disease 2019 (covid-19) situation report-101. 2020. https://www.who.int/docs/default-source/ coronaviruse/situation-reports/20200430-sitrep-101-covid-19. pdf?sfvrsn=2ba4e093 2 .

3 Simpson CR, Beever D, Challen K, et al, NIHR hibernated influenza studies collaborative group. The UK's pandemic influenza research portfolio: a model for future research on emerging infections. Lancet Infect Dis 2019:19:e295-300. doi:10.1016/S14733099(18)30786-2

4 Verity R, Okell LC, Dorigatti I, et al. Estimates of the severity of coronavirus disease 2019: a model-based analysis. Lancet Infect Dis 2020;0:S1473-3099(20)30243-7. doi:10.1016/S14733099(20)30243-7.

5 Fitzner J, Qasmieh S, Mounts AW, et al. Revision of clinical case definitions: influenza-like illness and severe acute respiratory infection. Bull World Health Organ 2018;96:122-8. doi:10.2471/ BLT.17.194514

6 WHO. WHO preliminary clinical description of severe acute respiratory syndrome. 2003.https://www.who.int/csr/sars/clinical/ en/.

7 Huang C, Wang Y, Li X, et al. Clinical features of patients infected with 2019 novel coronavirus in Wuhan, China. Lancet 2020;395:497506. doi:10.1016/S0140-6736(20)30183-5

8 Guan WJ, Ni ZY, Hu Y, et al, China Medical Treatment Expert Group for Covid-19. Clinical characteristics of coronavirus disease 2019 in China. N Engl J Med 2020;382:1708-20. doi:10.1056/ NEJMoa2002032

9 Chen N, Zhou M, Dong X, et al. Epidemiological and clinical characteristics of 99 cases of 2019 novel coronavirus pneumonia in Wuhan, China: a descriptive study. Lancet 2020;395:507-13. doi:10.1016/S0140-6736(20)30211-7

10 Onder G, Rezza G, Brusaferro S. Case-fatality rate and characteristics of patients dying in relation to covid-19 in Italy. JAMA 2020 doi:10.1001/jama.2020.4683

11 Richardson S, Hirsch JS, Narasimhan M, et al, and the Northwell COVID-19 Research Consortium. Presenting characteristics, comorbidities, and outcomes among 5700 patients hospitalized with covid-19 in the New York City area. JAMA 2020. doi:10.1001/ jama.2020.6775

12 Richardson S, Hirsch JS, Narasimhan M, et al. Clarification of mortality rate and data in abstract, results, and table 2. JAMA 2020. doi:10.1001/jama.2020.7681

13 Conolly A, Craig S. Health survey for England 2018: overweight and obesity in adults and children. 2019. https://files.digital.nhs.uk/52/ FD7E18/HSE18-Adult-Child-Obesity-rep.pdf.

14 Myles PR, Semple MG, Lim WS, et al, Influenza Clinical Information Network (FLU-CIN). Predictors of clinical outcome in a national hospitalised cohort across both waves of the influenza A/H1N1 pandemic 2009-2010 in the UK. Thorax 2012;67:709-17. doi:10.1136/thoraxjnl-2011-200266

15 Nguyen-Van-Tam JS, Openshaw PJM, Hashim A, et al, Influenza Clinical Information Network (FLU-CIN). Risk factors for hospitalisation and poor outcome with pandemic A/H1N1 influenza: United Kingdom first wave (May-September 2009). Thorax 2010;65:64551. doi:10.1136/thx.2010.135210

16 Alraddadi BM, Watson JT, Almarashi A, et al. Risk factors for primary Middle East respiratory syndrome coronavirus illness in humans, Saudi Arabia, 2014. Emerg Infect Dis 2016;22:49-55. doi:10.3201 eid2201.151340

17 Clohisey S, Baillie JK. Host susceptibility to severe influenza A virus infection. Crit Care 2019;23:303. doi:10.1186/s13054-0192566-7

18 Remuzzi A, Remuzzi G. COVID-19 and Italy: what next?Lancet 2020;395:1225-8. doi:10.1016/S01406736(20)30627-9

19 Grasselli G, Zangrillo A, Zanella A, et al, COVID-19 Lombardy ICU Network. Baseline characteristics and outcomes of 1591 patients infected with SARS-CoV-2 admitted to ICUs of the Lombardy Region, Italy. JAMA 2020. doi:10.1001/jama.2020.5394

20 ICNARC. ICNARC report on COVID-19 in critical care. 2020:1-9. https://www.icnarc.org/Our-Audit/Audits/Cmp/Reports.

21 ISARIC4C. ISARIC Coronavirus Clinical Characterisation Consortium. 2020. https://isaric4c.net/.

22 GOV.UK. New and Emerging Respiratory Virus Threats Advisory Group. 2020. https://www.gov.uk/government/groups/new-and-emergingrespiratory-virus-threats-advisory-group.

23 GOV.UK. Scientific Advisory Group for Emergencies. 2020. https:// www.gov.uk/government/groups/scientific-advisory-group-foremergencies-sage-coronavirus-covid-19-response.

24 GOV.UK. Scientific Pandemic Influenza Group on Modelling. 2020 https://www.gov.uk/government/groups/scientific-pandemicinfluenza-subgroup-on-modelling.

Web appendix: Supplementary material 1-online supplement

Web appendix: Supplementary material 2-protocol Web appendix: Supplementary material 3-case report form 